www.jmscr.igmpublication.org

Impact Factor 5.244

Index Copernicus Value: 83.27

ISSN (e)-2347-176x ISSN (p) 2455-0450

crossref DOI: _https://dx.doi.org/10.18535/jmscr/v4i10.45

\author{
Journal Of Medical Science And Clinical Research \\ IGM Publication \\ An official Publication of IGM Publication
}

\title{
Relationship between HbA1C and Left Ventricular Function in Type 2 Diabetes
}

\author{
Authors \\ Suganya $\mathbf{P}^{1}$, Senthilvelan $\mathbf{M}^{2}$, Resveen $\mathbf{H}^{3}$ \\ ${ }^{1}$ Postgraduate, ${ }^{2}$ Senior Professor, ${ }^{3}$ Assistant Professor
}

Department of Medicine, Rajah Muthiah Medical College and Hospital, Chidambaram, Tamilnadu, India

ABSTRACT
Diabetes mellitus is one of the leading public health problems and has profound effect on CVS. The risk of
cardiovascular complications is increased by twofold to six fold in subjects with diabetes.1The aim of this
study is to evaluate the association between left ventricular dysfunction and indexes of glycemic control-
glycosylated hemoglobin (HbAlC) in individuals with type 2 diabetes. Total 100 patients and 30 controls
were selected in this study and the relationship between HbAlC levels and LV function was obtained.

\section{INTRODUCTION}

The level of chronic hyperglycemia, as determined by measurements of glycosylated hemoglobin, may also be an independent risk factor for coronary heart disease. A $1 \%$ increase in levels of glycosylated hemoglobin (HbA1C) doubled the increase in cardiovascular disease. 4 HbA1C levels reflects the average blood glucose concentrations over a period of 2-3 months. The measurement is well standardized with less biological variability than plasma glucose levels. In this study, we examined the correlation between $\mathrm{HbA} 1 \mathrm{C}$ and left ventricular function in both diabetic and non-diabetic patients.

\section{MATERIALS AND METHODS}

This prospective clinical study was done among 100 diabetic patients attending the outpatient department of general medicine at Rajah Muthiah Medical College Hospital during the period of oct.2014 to aug.2016.Those patients in the age group of 40-60 yrs and who has been diagnosed as having diabetes according to $\mathrm{WHO}$ criteria were included in our study.HbA1C value $>6.5 \%$ is considered as diabetic. 3 A random sample of 30 non-diabetic patients whose age and sex were comparable to the study group was taken as control. Diabetic patients and control subjects having BP >140/90, s.cholesterol>240 mg/dl, history of smoking, alcoholism, heart and thyroid diseases, ECG changes and abnormal regional wall motions on 2D ECHO were excluded from the study. A detailed clinical history and complete general and systemic examination was taken. The following investigations were done; Resting 12 lead conventional ECG, HbA1c,S. cholesterol, S. triglyceride, Random blood sugar, Fasting blood sugar after an overnight fast, Post prandial blood sugar 2 hours after lunch, Chest $X$ ray PA. 2D Echocardiography was done on all subjects and the following assessed: LV systolic function was measured using ejection fraction (EF\%) the value $<55 \%$ was taken as index of systolic dysfunction. $\mathrm{LV}$ diastolic function was assessed by $\mathrm{VE}^{\mathrm{a}} / \mathrm{VA}^{\mathrm{b}}$ 
and the ratio $<0.75$ was taken as mild diastolic dysfunction, VE/VA 0.75 to 1.5 was taken as pseudonormal or moderate diastolic dysfunction and ratio $>1.5$ was considered severe diastolic dysfunction.a=Peak velocity of early LV filling (V max E);b=Peak velocity of late LV filling (V $\max A)$.

\section{OBSERVATION AND RESULTS}

Age and sex wise distribution of Diabetic cases

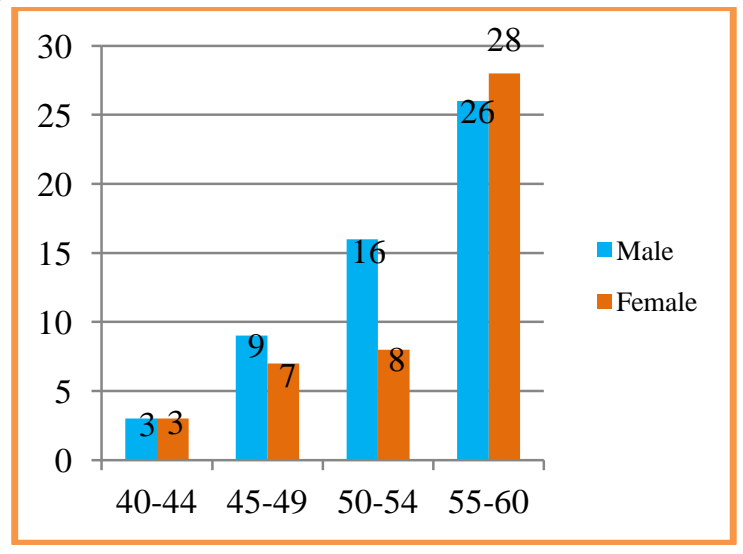

Comparison of HbA1C in Various Study

\begin{tabular}{|c|c|c|}
\hline \multirow{2}{*}{ Study } & \multicolumn{2}{|c|}{ HbA1C } \\
\cline { 2 - 3 } & Diabetes & Control \\
\hline${\text { Vinereanu et al }(\mathrm{n}=70)^{3}}^{3}$ & 8 & 5.5 \\
\hline${\text { Kyong et al }(\mathrm{n}=103)^{2}}^{2}$ & 7.62 & 5.3 \\
\hline Mohammed et al(n=80) $^{7}$ & 8 & 5.9 \\
\hline Present Study(n=130) & 7.68 & 5.56 \\
\hline $\mathrm{p}<0.0001(\mathrm{VHS})$ & & \\
\hline
\end{tabular}

Relationship between HbA1C \& LV Function in Diabetic Patients

\begin{tabular}{|c|c|c|}
\hline HbA1C & MEAN EF\% & MEAN Ve/Va \\
\hline $6.7-7.3$ & $52.95 \%$ & 0.785 \\
\hline $7.4-9.1$ & $45.25 \%$ & 0.97 \\
\hline$>9.1$ & $41.25 \%$ & 0.82 \\
\hline
\end{tabular}

Relationship between HbA1C and LV Function

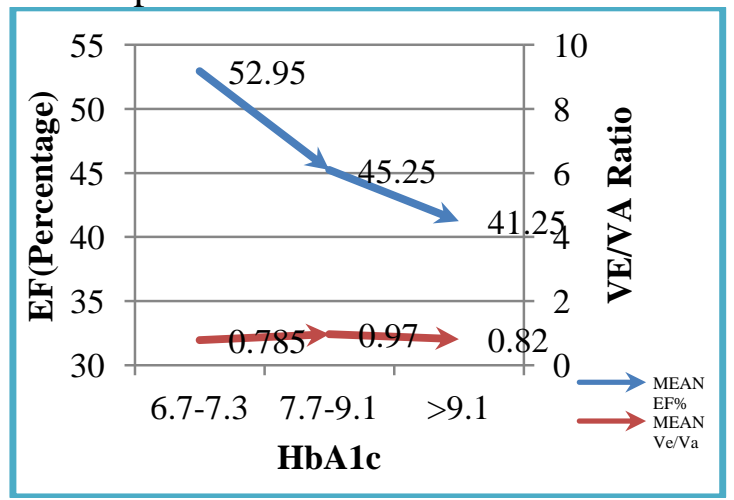

\section{DISCUSSION}

The commonest age group with diabetes in our study was 55-60 years age group in both diabetic male and females making up 54\% of all diabetic patients $(48.14 \%$ of all males and $60.8 \%$ of females).

In our study, mean value of $\mathrm{HbA1C}$ in diabetic was 7.68 as compared to 5.56 of control group. Mean value of $\mathrm{HbA1C}$ in study carried out by Kyong et $\mathrm{al}^{2}$ was 7.62 as compared to 5.3 of control group. This value is almost similar to our finding. Mean value of $\mathrm{HbA1C}$ in study by Vinereanuet $\mathrm{al}^{3}$, and Mohammed et $\mathrm{al}^{7}$ was 8 which was higher than control group.

Patients with HbA1c>9.1 had mean LVEF $41.25 \%$ as compared to $52.95 \%$ of patients with $\mathrm{HbA1c}<7.4$. Mean $\mathrm{Ve} / \mathrm{Va}$ was 0.785 in good glycemic control and towards mild diastolic dysfunction, while mean $\mathrm{Ve} / \mathrm{Va}$ of fair and poor glycemic control was 0.97 and 0.82 respectively which comes in pseudonormal range and suggests moderate diastolic dysfunction.

Studies carried out by Hameedullah et al $2009^{5}$ and Ali et al $2010^{6}$ also showed that with deterioration of glycemic control, left ventricular systolic and diastolic function deteriorates.

\section{CONCLUSION}

HbA1c is an independent risk factor for left ventricular systolic and diastolic dysfunction. Inverse relationship between $\mathrm{HbAlc}$ and $\mathrm{LV}$ function is demonstrated in this study.Hence HbA1C could be used as an important predictor for detecting left ventricular dysfunction in the early stage thereby preventing complications.

\section{REFERENCES}

1. Krall L.P., Richard S. Beaser Ed (Joslin's Diabetes manual, $12^{\text {th }}$ Ed., Pg. 130-35

2. Kyong et al Korean circulation $\mathbf{J}$ 2006;36:411-417 (ISSN 1738-5520)

3. Vinereanu et al clinical science (2003) 105,591-599

4. Joslin Diabetes Mellitus, $14^{\text {th }}$ Ed. 976-992 Saunders J, Mathewkutty S, Drazner MH, McGuire DK: Cardiomyopathy in type 2 
diabetes: Update on pathophysiological mechanisms. Herz 33:184, 2008.)

5. Endocrine Abstracts (2010) 22 P349

6. Acta Diabeloca Volume 44, Number 4, 193-200

7. Mohammed et al.2009 jam coll cardiol,2009;54;1524-1532.doi 101016 j:jace 2009.04.074 